\title{
NiÑOS, NUEVAS TECNOLOGÍAS Y GÉNERO: HACIA LA DEFINI- CIÓN DE UNA AGENDA DE INVESTIGACIÓN
}

\section{Children New technologies and Gender: Towards the Definition of a Research Agenda}

\author{
Dra. Carolina Duek \\ Investigadora del CONICET, Profesora de la Universidad de Buenos Aires, Argentina. \\ E-Mail: duekcarolina@gmail.com \\ (1D http://orcid.org/0000-0002-3103-0363
}

\section{Dr. Sebastián Benítez Larghi}

Investigador del CONICET, Profesor de la Universidad Nacional de La Plata, Centro Interdisciplinario de Metodología de las Ciencias Sociales (CIMeCS), Instituto de Investigaciones en Humanidades y Ciencias Sociales, Departamento de Sociología, La Plata, Argentina.

E-Mail: sebastianbenitezlarghi@gmail.com

(1D) http:/ / orcid.org/0000-0002-2646-0035

\section{Dra. Marina Moguillansky}

Investigadora del CONICET y Profesora de la Universidad Nacional de San Martín, Argentina.

E-Mail: mmoguillansky@gmail.com

(1D http://orcid.org/0000-0002-8873-1136

Fecha de recepción del artículo: 26/09/2017

Fecha de aceptación definitiva: 19/01/2017

\begin{abstract}
RESUMEN
La relación entre las Tecnologías de la Información y de la Comunicación (TIC) con diversos aspectos de la vida cotidiana contemporánea constituye un fructífero campo dentro de los estudios sociales. Las constantes indagaciones sobre las formas de uso, de socialización, de significación y de representación ubican a las TIC como centro de grandes interrogantes de la investigación en ciencias sociales en el siglo XXI. Este artículo tiene como objetivo componer una agenda para la investigación de las TIC en relación con el género, los niños, las niñas y los juegos. Si bien estos ejes han sido estudiados, las intersecciones y las articulaciones que identificamos en los últimos proyectos nos abrieron nuevas perspectivas para la indagación. ¿Qué hacen los niños y las niñas con los dispositivos con los que se vinculan? ¿De qué formas el género se constituye como una variable interviniente en las dinámicas, personajes, acciones y roles que se pueden (o no) asumir durante las interacciones lúdicas? En el artículo se presentan los diferentes elementos de una agenda de trabajo con los principales ejes, problemas, hipótesis y metodologías sobre las cuales se proyectan nuestros trabajos actuales y futuros ${ }^{1}$ como forma de contribuir al campo en el que se enmarcan.
\end{abstract}

Palabras clave: Infancias; Género; Dispositivos; Juegos; Trayectorias.

\footnotetext{
1 Aquí se presentan algunos resultados de diversos proyectos de investigación dirigidos por los autores (PIO FLACSOCONICET 'Niñez, género y TIC. Un estudio de las tecnobiografías de niños y niñas en la Argentina' y PICT 2014-3277 ‘TIC, juventudes e inclusión social. Una evaluación cualitativa del impacto del Programa Conectar Igualdad en la trayectoria educativa, laboral, social y política de jóvenes egresados de la escuela secundaria en La Plata y Gran La Plata’).
}

(C) Ediciones Universidad de Salamanca / CC BY - NC ND Fonseca, Journal of Communication, n. 14, 2017, pp. 167-179 


\begin{abstract}
The relationship between information and communications technology (ICT) and a number of aspects of contemporary everyday life constitutes a productive field inside cultural studies. The constant enquiries about uses, socialization and representations place ICT in the midst of Social Sciences research in the 21 st century. This article aims to compose a research agenda for future research on ICT related to gender, children and play. All those axes have been independently analyzed, but the intersections and articulations we found in our research open new perspectives to question. What do children do with the devices? In which ways, does gender constitute an intervening variable in the dynamics, characters, actions and roles that may (or may not) be assumed during games? This article presents a series of dimensions that constitute a possible research agenda organized by the main axes, problems, hypotheses and methodologies on which our actual and future research is projected as a way of contributing to the field in which they are framed.
\end{abstract}

Key words: Childhoods; Gender; Devices; Play; Trajectories.

\title{
1. INTRODUCCIÓN
}

En las sociedades occidentales, el género constituye un sistema de asignación de roles, derechos y responsabilidades, a través de los procesos de socialización y generización, que clasifican y distribuyen a hombres y mujeres en el espacio social (Bourdieu, 1998; Ridgeway, et al., 2009). El género funciona como sistema de estratificación, puesto que condiciona el acceso a recursos y capitales (Risman y Davis, 2013). En occidente la ideología dominante afirma que las diferencias y las desigualdades de género derivan de un sustrato biológico, es decir, de las diferencias entre los sexos (Lorber, 1994) y, de esta forma, se naturalizan y legitiman las desigualdades entre varones y mujeres.

En este marco, la emergencia y amplia difusión social de las Tecnologías de Información y Comunicación (TIC) abre nuevos interrogantes en torno al modo en que las diferencias de género se procesan a través de la apropiación de las tecnologías, contribuyendo a reproducir y/o ampliar desigualdades existentes, pero también habilitando usos novedosos que tensionan los roles de género instituidos, cuestión que se manifiesta de modo evidente en torno a los juegos y a otras prácticas significativas de las infancias contemporáneas.

Diversos estudios afirman que, lejos de ser neutrales, las TIC están generizadas desde la producción y el diseño de objetos, espacios y contenidos hasta la manera de dirigirse a los públicos a los que -como representación- están orientadas (Hafkin y Taggart, 2001). Livingstone y Helsper (2007) sostienen que es poca la evidencia empírica que tenemos acerca de las desigualdades de género en términos del acceso y la demanda de uso de TIC: sabemos poco sobre las razones por las cuales la mayoría de las mujeres, en comparación con los varones, usan menos las TIC. Y es por ello que este artículo se proyecta como una propuesta teórica sobre el tema que se traduce, más adelante, en un abordaje metodológico.

Un obstáculo para construir conocimientos ha sido la creencia de que los niños configuran la llamada «generación Internet» y son percibidos como expertos en cuestiones tecnológicas (Livingstone y Helsper, 2007, p. 672). En este sentido, la metáfora de nativos digitales (Prensky, 2001) ha contribuido a fomentar miradas en torno a la niñez que tienden a presentarla como un universo homogéneo que se distingue por su capacidad para aprender a usar las TIC. Livingstone y Helsper (2007) indican que las diferencias de género en la apropiación de TIC persisten, incluso cuando el acceso hogareño es equiparable. Señalan que, en cierto momento entre la niñez y la adolescencia, las intensidades de uso, así como las posibilidades de extender los intereses y conocimientos empiezan a diferenciarse entre varones y mujeres. Un ejemplo de estas diferenciaciones puede observarse en juegos como el GTA (Grand 
Theft Auto), uno de los videojuegos más vendidos de la historia ${ }^{2}$. Al respecto, preguntamos a un conjunto de diez niñas entre siete y diez años (en el marco de una investigación, ver Duek, 2014) sobre el GTA y todas estuvieron de acuerdo en que ese juego «no era para ellas» que «es para varones», «es muy de matar», entre otros comentarios tajantes que parecían excluir de su universo de juegos al GTA por no ser «para ellas».

Este artículo tiene como objetivo componer una agenda para la investigación de las TIC en relación con el género, los niños y niñas y las nuevas tecnologías. Una agenda (Leona, 2014) supone la articulación de temas, problemas, jerarquizaciones, omisiones y relevancias que rodean un problema específico. Una agenda de investigación supone la combinación de disciplinas, herramientas, miradas y problemas: esa es aquí, a la vez, nuestra apuesta y propuesta.

Abordaremos, en algunos pasajes, los juegos contemporáneos como ejemplo (el juego es una dimensión crucial de la vida de los niños y de las niñas, Duek, 2014) y presentaremos algunos resultados de investigaciones en curso. Si bien los ejes han sido muy estudiados de manera independiente, las intersecciones y las articulaciones que identificamos en los últimos proyectos que dirigimos nos abrieron nuevas perspectivas para la indagación. ¿Qué hacen los niños y las niñas con los dispositivos con los que se vinculan para jugar? ¿De qué formas el género se constituye como un factor que moldea las dinámicas, personajes, acciones y roles que se pueden (o no) asumir durante las interacciones lúdicas? Sostenemos que el estudio del vínculo entre el género, la niñez, las TIC y los juegos debe hacerse siempre de manera situada y contextualizada (es decir, desnaturalizando la relación entre las TIC y los sujetos) y teniendo en cuenta las representaciones, imaginarios y estructuras ideológicas de los artefactos socio-técnicos y de los contenidos construidos en y a través de las TIC. En consecuencia, en la Sección 3, la agenda propone como objetivo principal de investigación comprender los complejos y diferenciales procesos de apropiación más que medir brechas digitales entre géneros. Cuestión que metodológicamente obliga a recuperar la perspectiva de los actores mediante una estrategia de corte cualitativo tendiente a reconstruir las trayectorias individuales y familiares que permita un análisis comparativo para detectar los mecanismos por los cuales las diferencias -y desigualdades- entre mujeres y varones se cristalizan en el uso de las TIC y, más particularmente, en las prácticas lúdicas.

\section{ANTECEDENTES Y MARCO TEÓRICO: CONTRIBUCIONES CLAVE PARA ABORDAR EL JUEGO CONTEMPORÁNEO EN CLAVE DE GÉNERO}

Resulta difícil encontrar abordajes que combinen preocupaciones sobre juego y género de manera sostenida y articulada. Es por ello que propondremos, en este trabajo, algunas claves para pensar el vínculo recorriendo algunos aportes teóricos clave sobre la relación entre los niños, las niñas y las TIC de forma general y, en particular, las formas en las que se intersectan las preocupaciones analíticas sobre el juego y el género.

Uno de los grandes acuerdos de la bibliografía sugiere que al abordar los vínculos entre género y TIC se registran estereotipos que asignan usos y habilidades específicas para varones y mujeres (Cassell y Jenkins, 2000; Livingstone y Haddon, 2012, entre otros). Estos estereotipos tienden a consagrar diferencias de género culturalmente dominantes, moldeando la experiencia de varones y mujeres respecto a las tecnologías en general, y a las TIC en particular, en las distintas etapas vitales. Las categorías de «juegos de varón» y «juegos de nena» aparecen como grandes organizadoras de las prácticas lúdicas, sobre todo en las últimas décadas. Esta construcción cultural (que nada tiene de biológica ni

\footnotetext{
2 Solo el GTA V vendió 52 millones de copias en todo el mundo, un récord inimaginable para sus productores: http://www.gamereactor.es/noticias/232914/Ventas+GTA+V+suma+52+millones+de+copias+expendidas.
}

(C) Ediciones Universidad de Salamanca / CC BY - NC ND Fonseca, Journal of Communication, n. 14, 2017, pp. 167-179 
determinada) constriñe, a su vez, la relación de los más chicos con las nuevas tecnologías. Al respecto, Tomte (2008) sostiene que los varones de escuela primaria se muestran más confiados respecto de sus habilidades digitales que las mujeres, mientras que Volman et al. (2005) señalan que las niñas se sienten menos cómodas que los niños al usar las TIC y que estos se autoperciben como más expertos incluso que sus maestros.

Plantearemos aquí tres grandes ejes que, en función de nuestra experiencia como investigadores, consideramos centrales para toda investigación cualitativa sobre juego, género y TIC. Esta propuesta no es definitiva ni abarca todas las dimensiones posibles, sino que está en constante reformulación con cada interrogante o variable que aparece en los proyectos y en los trabajos de campo. Proponemos, entonces, tres ejes que nos permiten comenzar a pensar todos los procesos de investigación de manera abierta, productiva y reflexiva.

Un primer eje para abordar una investigación que vincule a niños, niñas y el género en función de los juegos y de las pantallas es la desnaturalización de la relación entre las TIC y los sujetos (Duek, 2014). Considerar la relación de los sujetos con las TIC como una construcción nos permite desplegar un análisis que atiende a la configuración social, económica, cultural y educativa de las formas en las que se estructuran las representaciones hegemónicas sobre el género y el juego. De ese modo, la identificación de los condicionamientos, de las posibilidades y de las tensiones existentes en(tre) los sujetos sociales, nos abre un abanico que nos informa, en profundidad, las formas en las que cada sujeto accede, usa, construye y se apropia de ciertos repertorios de juegos mediados tecnológicamente.

Uno de los aportes más importantes al respecto es el de Cassell y Jenkins (1998) y el de Kafai et al. (2008). Este equipo de investigación dedicado a los videojuegos y al género publicó dos libros centrales para este campo con diez años de diferencia. El primero, From Barbie to Mortal Kombat. Gender and computer games, es probablemente el primer aporte sistemático y profundo sobre la relación entre los más chicos y los juegos en clave de género. Cassell y Jenkins presentan diferentes ejes y análisis que permitieron la construcción de un campo de estudios. Las reivindicaciones de «movimientos de chicas» que comienzan a diseñar y a pensar su rol en y para los juegos nos indica no solo una conciencia sobre las tensiones y desigualdades a combatir, sino también estrategias a través de las cuales la creatividad, el ingenio y la especialización en TIC apuntan a desarticular las representaciones (ver también Turkle, 2011). Diez años después, en Beyond Barbie and Mortal Kombat. New perspectives on gender and gaming, se presentan experiencias sostenidas en el tiempo y nuevos espacios desde los que se proponen desarmar y reconstruir las tramas de género vinculadas a los juegos. Las inclusiones, las formas de participar y de colaborar en juegos en red aparecen como pequeñas conquistas de un largo camino por recorrer. La clave que encontramos en ambos libros es, a la vez, compleja y sencilla: la relación entre el género y las TIC es una construcción que se cristaliza en los usos de los videojuegos y es por ello que debe ser puesta en cuestión y reconstruida en función de la perspectiva de los actores en (y del) juego. No hay juegos «de niñas» ni «de niños» sino construcciones hegemónicas que los clasifican de ese modo.

Un segundo eje se vincula con la necesidad de desarrollar análisis críticos de la estructura ideológica de los contenidos de los medios de comunicación, los programas informáticos, los contenidos digitales, los videojuegos, así como de sus estereotipos y de los modelos de conducta que suponen. La orientación de género dominante en la cultura, en los medios, en los discursos de los adultos, interviene en los modos en que niños y niñas construyen sus identificaciones como sujetos y se apropian de las TIC (Benítez Larghi, et al., 2013). Lo que es fundamental para abordar la relación entre niños, niñas y representaciones de prácticas y juegos, es caracterizar críticamente las maneras en las que los más chicos, representados en las pantallas, interactúan, consumen, se vinculan, disputan espacios y

(C) Ediciones Universidad de Salamanca / CC BY - NC ND Fonseca, Journal of Communication, n. 14, 2017, pp. 167-179 
distribuyen el poder. La representación de los niños como sujetos autónomos, independientes del mundo adulto, capaces de resolver sus necesidades y deseos (ver Duek, 2013), ubica a los más chicos en el terreno de la demanda, de los pedidos de compra que son, finalmente, el punto de pasaje necesario para la reproducción (y para el aumento de ventas, claro) de los productos que se ofrecen en las publicidades y en los objetos derivados de los personajes de los programas de televisión más vistos (desde mochilas y útiles escolares pasando por vestimenta, juegos de mesa reconvertidos, revistas, stickers, solo por mencionar algunos de los tantos objetos disponibles). Pero más allá de las representaciones, los modos en que los niños se relacionan con las pantallas y las tecnologías, tienen una estrecha relación con el mundo adulto que establece tiempos, reglas y espacios (la omisión es, también, una forma de establecer tiempos y reglas).

Ver televisión ya no alcanza: se trata de construir entramados de consumo que permitan que los más chicos, en un momento de multiplicación de la oferta en múltiples soportes, sigan en contacto con sus personajes y programas de televisión (Jenkins, 2005). Pero, también, se despliega una dimensión social en la selección de preferencias, de personajes y de programas porque «el espectador se suma a un público potencialmente inmenso y anónimo que mira simultáneamente, y mantiene con él un vínculo invisible, es una clase de common knowledge» [conocimiento común] (Wolton, 1995, p. 126). El carácter productivo del texto [televisivo] depende del contexto, su naturaleza y su posibilidad de ser recibido (Morley, 1997).

Finalmente, el tercer eje que proponemos para la construcción de investigaciones se vincula con el análisis de las trayectorias sociales y culturales en las que se tejen apropiaciones diferenciales de las TIC y los posibles abordajes sobre ellas. Algunos autores señalan que, más allá del uso más intensivo que hacen los varones de la computadora, existen usos y habilidades diferenciales (no solo usan más las tecnologías, sino que desarrollan diferentes destrezas). Según esta perspectiva, los varones tienden a jugar y a programar, mientras que las mujeres tienen mayor protagonismo en las redes sociales virtuales y hacen usos más creativos de la tecnología (Howe y Mercer, 2007).

En la misma línea, Volman et al. (2005) encuentran que las mujeres muestran entusiasmo en aplicaciones como los procesadores de texto y los programas para dibujar. El proceso de aprendizaje también tiende a ser diferente entre chicos y chicas adolescentes y jóvenes. Según la literatura, las últimas prefieren la mediación de un adulto experto, mientras los varones prefieren aprender por sí mismos o a través de amigos. Como puede apreciarse, mucho se conoce respecto a las diferencias existentes en relación a las prácticas, actitudes y representaciones de las TIC entre las y los adolescentes. Sin embargo, lo que aún no se ha podido conocer es cuándo y cómo comienza esta diferenciación (Tomte, 2008, pp. 6-7). Es por ello que consideramos crucial la reconstrucción de las trayectorias de apropiación de las TIC de niños y niñas, porque es allí donde se pueden identificar las formas en las que se estructuran las apropiaciones, los intereses y las maneras en las que cada una de las biografías se imbrica con otras. El rol de los adultos, las experiencias compartidas (o no), la incidencia de los pares con los que se comparte la cotidianeidad son algunas de las claves para identificar la genealogía de los usos que los sujetos hacen de las TIC.

El rol de los juegos entre pantallas en cualquiera de las plataformas, redes sociales o páginas va a tener su correlato si se articula con una historia de los vínculos que lo sostienen. Jugar, como dijo Huizinga (1938), es mucho más que un juego, porque condensa las particularidades culturales de un tiempo en la historia de la humanidad. En la actualidad, jugar se ha complejizado no solo por la circulación de discursos que enriquecen los juegos (en el sentido de Caillois, 1967), sino por las múltiples plataformas y dispositivos electrónicos en los que se pueden encontrar propuestas lúdicas (o reconstruirlas). Los tres ejes que proponemos para la construcción de investigaciones cualitativas vinculadas 
con el juego y el género se vinculan con la desnaturalización, el análisis crítico y la reconstrucción de las trayectorias de los sujetos sociales.

\section{Metodología: ejes para LA ESTRUCturación DE LA AGENDA DE INVESTIGACIÓN}

Dentro del campo de los estudios sociales de la ciencia, tecnología y sociedad, las desigualdades de género en la relación con las TIC constituyen un foco de constante interés, debate e intervención. Tanto la literatura existente como nuestras propias investigaciones previas coinciden en señalar que, a partir de la adolescencia, se consolidan ciertos patrones desiguales en el acceso y usos de las TIC entre varones y mujeres, siendo las diferencias en relación a los videojuegos una de sus manifestaciones más elocuentes. Estas modalidades generizadas de relación con las tecnologías podrían condicionar las posteriores trayectorias educativas, así como las inserciones laborales y profesionales. Es así que la difusión generizada de las TIC contribuye a la emergencia de nuevos tipos de desigualdades sociogenéricas. En este contexto, es necesario indagar los procesos de construcción de trayectorias generizadas de acceso a las TIC desde la niñez, a través de las socializaciones múltiples que ocurren en el marco de la familia, la escuela, el consumo de mercancías y de narrativas/mensajes mediáticos. De allí que entendamos que uno de los objetivos principales de la investigación social sobre estos temas debe apuntar a comprender el modo en que las desigualdades de género y los procesos de apropiación tecnológica se co-constituyen a partir del estudio comparativo de las trayectorias de apropiación de las TIC, en especial de sus vínculos con los video-juegos, por parte de niñas y niños en las sociedades contemporáneas.

En un artículo reciente, Jenson y Castell (2010) revisaron un corpus significativo de investigaciones sobre género y videojuegos, identificando tres tipos de sesgos. El primer sesgo consiste en una desconsideración del género más allá del sexo o en la confusión de ambos, que impide percibir los procesos de identificación, las normas sociales y los roles de género en su dimensión construida y convencional. Este sesgo proviene de una ausencia de reflexión teórica sobre el género en tanto construcción social e histórica y conduce a perspectivas biologicistas que tienden a naturalizar las diferencias entre hombres y mujeres, niños y niñas. En segundo lugar, hay un sesgo que resulta del intento de identificar patrones específicos de juego y preferencias que serían propias del género femenino, para luego promoverlos con el objetivo de lograr mayor equidad. Este tipo de investigación invisibiliza las diferencias al interior, es decir, entre las propias mujeres y niñas, obviando las distintas posiciones subjetivas que pueden identificarse como femeninas sin necesariamente converger en otras características (como, precisamente, las habilidades o gustos en materia de TIC). El tercer sesgo se encuentra en investigaciones que se ocupan del género solo para mostrar que no es una variable significativa, obturando toda reflexión posterior. La dificultad consiste, en este tipo de trabajos, en la ausencia de una problematización acerca de qué significan las convergencias encontradas, cómo se construyen y qué otras diferencias, quizás, se ocultan en los datos que se obtienen.

Nuestra propuesta evita estas dificultades teórico-metodológicas para construir conocimientos sistemáticos y a la vez sensibles a las particularidades subjetivas de las relaciones de niños y niñas con las TIC. Para ello resulta indispensable complejizar la forma de concebir la relación entre sujetos y dispositivos a partir del concepto de «apropiación» de las tecnologías. Como sostiene Winocur (2009), la apropiación de las tecnologías es un proceso que se construye sobre la base de experiencias presentes y pasadas con otras tecnologías, así como de expectativas, nociones previas e ideas sobre estos artefactos tanto individuales como familiares y colectivas, y en el marco de entramados culturales en lo que se les otorga cierta valoración y significación. En este proceso, cómo se usan los artefactos es

(C) Ediciones Universidad de Salamanca / CC BY - NC ND Fonseca, Journal of Communication, n. 14, 2017, pp. 167-179 
indisociable de cómo se los significa y representa (Cabrera Paz, 2009). Como la dimensión del sentido es constitutiva de la apropiación de las TIC, consideramos indispensable partir de un paradigma cualitativo e interpretativo de la investigación social (Vasilachis de Gialdino, 1992 y Taylor y Bogdan, 1986), que resulta adecuado para el recorte empírico de los cruces entre niñez, juegos y TIC. El paradigma cualitativo asimismo supone un tipo de investigación que se construye en un vínculo dialógico de los investigadores y los sujetos (Scribano, 2008). Esta apertura dialógica y la construcción de datos a partir del encuentro con los sujetos permite captar la diversidad de posiciones de género y de formas de apropiación de las tecnologías, sin prefijar las categorías ni obligar a la complejidad a reducirse al sistema binario del género.

En este contexto, proponemos dos grandes instancias que son, a la vez, teóricas y metodológicas para abordar las problemáticas de forma cualitativa y en profundidad: la reconstrucción de las trayectorias de apropiación de TIC y su posterior comparación en clave de género.

\section{LA RECONSTRUCCIÓN DE LAS TRAYECTORIAS DE APROPIACIÓN DE TIC}

Dentro de este enfoque, entonces, un primer paso en la investigación cualitativa supone la reconstrucción de las trayectorias de acceso y usos de TIC de niños y niñas y sus familias en el hogar, el tiempo libre y la escuela. En relación al hogar deben atenderse las características de los primeros contactos con TIC (edad, lugar de uso, referentes), las condiciones de acceso y conectividad en el hogar, la adquisición de dispositivos propios, las modalidades de uso, reglas y límites, los procesos de aprendizaje de habilidades informáticas y las representaciones e imaginarios de los padres u otros adultos significativos acerca de las relaciones entre género y TIC. Estas dimensiones se articulan por los sitios preferidos para jugar en línea o para contactarse con pares y familiares a través de ellos (por ejemplo, Facebook, Club Penguin, Mundo Gaturro - de gran repercusión en América Latina- entre tantos otros) ${ }^{3}$. Un abordaje de la vida cotidiana permite profundizar las prácticas y los significados que tienen, para los sujetos, dichas acciones.

En relación al tiempo libre, esta exploración se realiza considerando, como ejemplo ordenador, al juego en todas sus variantes intersectándolo con el contacto con grupos de pares y los consumos culturales. Comprender y analizar los hábitos lúdicos mediados por TIC en niños y niñas deviene una herramienta crucial para la investigación social, porque permite profundizar diversos ejes: las representaciones de roles de género en los juegos mediados por TIC, los imaginarios y estereotipos sobre las tecnologías y el género en el grupo de pares y los imaginarios sobre géneros y tecnologías en las narrativas mediáticas consumidas por niños y niñas (según referidas por ellos mismos).

Por último, es preciso analizar los procesos de socialización en y a través del uso de TIC en la escuela teniendo en cuenta las condiciones de acceso a TIC y conectividad de cada institución de pertenencia; las habilidades informáticas del personal del entorno escolar; las modalidades institucionales y curriculares de uso de TIC; las representaciones de los maestros sobre TIC y usos escolares. Por ejemplo, muchas instituciones utilizan, para las primeras aproximaciones, los juegos en línea para construir estrategias de aprendizaje. Este tipo de decisiones institucionales van a intervenir de manera crucial en las apropiaciones y usos de sus alumnos. La escuela sigue siendo el lugar en donde se tejen las tramas de socialización que van a intervenir de manera crucial en los usos y apropiaciones de las TIC en momentos de ocio y juego.

Para esta primera instancia, realizamos historias de vida. Esta técnica implica la utilización de guiones flexibles de entrevista, en encuentros reiterados con los niños y las niñas y sus familiares o

\footnotetext{
${ }^{3}$ Las páginas mencionadas son: www.facebook.com; www.clubpenguin.com/es; www.mundogaturro.com

(C) Ediciones Universidad de Salamanca / CC BY - NC ND Fonseca, Journal of Communication, n. 14, 2017, pp. 167-179
} 
referentes directos. Estos guiones permitirán enfocar los relatos en los eventos significativos de las trayectorias de relación con la computadora, la telefonía celular, las consolas de videojuegos y las tabletas, con conexión a Internet. Es importante señalar que, aunque el término trayectoria pueda sugerir una idea de recorrido lineal en un uso creciente y progresivo de tecnologías, siguiendo a Berteaux (2005) y basados en nuestra propia experiencia en investigaciones biográficas previas podemos indicar que el vínculo de las personas con los juegos y las TIC, e incluso en sus identificaciones de género, sigue derroteros cambiantes y no lineales.

En particular, optamos por un tipo de historia de vida orientada a los temas que se indagan. En tal caso, los guiones de entrevista, si bien tuvieron cierta apertura y fueron flexibles para incorporar los datos emergentes de la interacción, se construyeron con una cierta orientación hacia determinadas prácticas, representaciones y motivaciones. Debido a que el foco de nuestra área de indagación es el vínculo de los sujetos con las TIC, que se encuentra altamente naturalizado y encarnado en las prácticas de la vida cotidiana, hemos detectado tanto en la literatura existente como en nuestras investigaciones, que resulta necesario y provechoso orientar las preguntas y los relatos de los sujetos para que aparezcan las tecnologías en toda su ubicuidad. Por ello sugerimos que una estrategia metodológica adecuada es la construcción de tecnobiografías (Ching y Vigdor, 2005). En efecto, la construcción de tecnobiografías de niños, dentro de sus contextos familiares es una alternativa muy promisoria para estudiar los procesos socialmente moldeados de interacción con las TIC. Este tipo de estrategia metodológica permite atender a construcción histórica de las interacciones entre género, clase social y desigualdades geográficas, es decir, permite observar el aspecto procesual del fenómeno de la interseccionalidad ${ }^{4}$.

La historia de vida de Ignacio, alumno de una escuela media de la localidad de Berisso, nos permite comprender el rol de la apropiación de las TIC en la configuración de procesos de igual$\mathrm{dad} /$ desigualdad. Al momento de realizar las primeras entrevistas, Ignacio tenía diecisiete años. Desde su nacimiento vivía con su madre y su hermana dos años mayor. Ignacio comenzó a jugar a los jueguitos a los cinco años. Al principio no había computadora en su casa, por lo que jugaba Mario Bros. con una consola Sega, alquilando los juegos o iba a jugar con otros amigos a la casa de una amiga de su hermana, que fue la primera del círculo de amigos en tener computadora propia. Luego, esta familia amiga quiso actualizar su computadora, por lo cual la madre de Ignacio logró comprarle su anterior PC a un precio económico («era la única manera de poder comprar una compu para nosotros, aunque no se trataba de una máquina muy nueva», dice Ignacio). Con esta computadora en su hogar, Ignacio continuó jugando e investigando, ya que impulsado por su madre siempre fue muy curioso con la tecnología. A uno de sus mejores amigos Ignacio lo conoció en el ciber del barrio ${ }^{5}$. Ignacio tenía nueve años y pasaba todas las tardes ahí. Dos o tres horas por día, jugando en la computadora. Solo dejó de ir al ciber cuando comenzaron a tener Internet en su casa, lo que ocurrió cuando tenía alrededor de doce años. Al momento en que se realizaron las primeras entrevistas, Ignacio era un usuario asiduo de YouTube. Miraba un gran número de videos cotidianamente, sigue a ciertas personas que publican video-blogs regularmente. Él mismo había filmado «un cover de batería», que luego subió a su propio canal de YouTube que felizmente, para el orgullo y divertimento de Ignacio, alcanzó trescientas visitas

\footnotetext{
${ }^{4}$ De acuerdo con La Barbera (2010: 63), la noción de interseccionalidad se refiere a los procesos -complejos, irreducibles, variables- que en cada contexto derivan de la interacción de factores sociales, económicos, políticos, culturales y simbólicos.

${ }^{5}$ Ciber o cibercafé es el nombre asignado en Argentina a los espacios de alquiler temporal de computadoras para acceder a juegos on line o a Internet y diferentes espacios de conectividad. El acceso a Internet través de los cibercafés fue un fenómeno muy relevante en Argentina y otros países durante la década de 2000, disminuyendo su centralidad hacia 2010 hasta casi desaparecer en el transcurso de los últimos años por la presencia de los teléfonos celulares que abarataron y facilitaron la conexión.
} 
y reproducciones. En 2016, al contactarlo para nuevas entrevistas, nos enteramos de que Ignacio había ingresado a la carrera de Informática en la UNLP.

La historia de Ignacio permite reflexionar acerca de cómo el abordaje de la apropiación en función de las trayectorias permite comprender mejor el sentido de los usos de las tecnologías digitales e Internet. La literatura dominante sobre TIC y desarrollo ha puesto el énfasis en las llamadas brechas digitales. En primera instancia, se pensó las desigualdades digitales en términos del acceso/no acceso a dispositivos y conexiones. Luego, con la masificación de ciertos equipos (especialmente los teléfonos celulares), el foco se desplazó al análisis de los usos y aprovechamientos de la tecnología. En ese punto, se construyeron algunas categorías para clasificar -y calificar- los tipos de usos. Así, muchos autores han distinguido usos productivos de las TIC (para estudiar y trabajar) de improductivos (para entretenerse y comunicarse con amigos). Así se estaría generando una brecha ya no por la capacidad de acceso, sino por la calidad de los usos que se le da a la tecnología: quienes desarrollen el primer tipo de uso acumularían ventajas respecto a quienes solamente se queden en el segundo tipo de usos, generando así nuevas desigualdades. Pues bien, estos estudios tienden a sacar fotografías (siempre estáticas) y perder de vista las trayectorias. Si se valorara la experiencia de Ignacio únicamente en función de los usos desarrollados durante su infancia y su adolescencia, estos hubiesen sido considerados improductivos. La literatura dominante $-\mathrm{y}$ su metodología cuantitativa- hubiese categorizado a Ignacio como «pobre digital». Sin embargo, su presente como estudiante de Informática permite sospechar que aquellos usos implicaban mucho más que su objetivo evidente. Sin dudas, a lo largo de su vida Ignacio fue desarrollando habilidades, intereses y deseos en torno a la informática que luego contribuyeron a configurar su pasaje de la escuela secundaria a la vida universitaria. En definitiva, solamente abarcando las trayectorias de apropiación pueden comprenderse los vínculos entre tecnología y desigualdades leyéndolos no meramente como una posición/condición estanca en un determinado momento sino como fruto de un conjunto complejo de procesos de acumulación de ventajas y desventajas.

\section{LA COMPARACIÓN DE TRAYECTORIAS EN CLAVE DE GÉNERO}

Un segundo paso de la investigación consistió en comparar sistemáticamente en clave de género las trayectorias de apropiación de TIC por niñas y niños en los tres ámbitos indicados más arriba (el hogar, el tiempo libre y la escuela) atendiendo a las intersecciones del género con la clase social. Las chicas de familias de sectores populares tienen en promedio un primer acceso a la computadora bastante tardío que se ubica alrededor de los 12 o 14 años y ocurre, por lo general, en un cibercafé. El proceso de aprendizaje del uso de las TIC tiene lugar en la escuela a través de la educación formal, y en menor medida, por la experiencia personal en cibercafés. Las jóvenes de sectores populares accedieron también tardíamente a sus primeras computadoras personales, en la mayoría de los casos a través de políticas públicas como el Programa Conectar Igualdad ${ }^{6}$. Sus habilidades y destrezas se concentran en los usos comunicativos y expresivos de la computadora. Los varones de sectores populares tienen un acceso algo más temprano que las mujeres del mismo origen socioeconómico, y se observa una mayor variabilidad: el primer uso de la computadora puede registrarse tan pronto como a los 8 años de edad, pero en algunos casos se demora hasta los 12 años. En casi todos los casos ese primer contacto tuvo lugar en cibercafés, conformando así una cierta experiencia de clase se intersecta con el

\footnotetext{
${ }^{6}$ El PCI consiste en un modelo 1 a 1 de incorporación de TIC a la educación de carácter universal. Es decir, que provee una netbook a todos los estudiantes de escuelas públicas secundarias en la Argentina. El PCI ha sido implementado conjuntamente por los Ministerio de Educación y de Planificación, la Jefatura de Gabinete y la Administración Nacional de Servicios de Seguridad Social (ANSES). Hacia fines de 2015 se habían entregado más de cinco millones de netbooks en todo el país, según los datos consignados en http://www.conectarigualdad.gob.ar.
}

(C) Ediciones Universidad de Salamanca / CC BY - NC ND Fonseca, Journal of Communication, n. 14, 2017, pp. 167-179 
género, ya que las mujeres frecuentan menos estos espacios. Confirmando lo que señalaba la literatura al respecto, las estrategias de aprendizaje de varones y mujeres difieren, ya que ellas prefieren la mediación de un adulto, mientras que el aprendizaje de los varones se realiza a través de la exploración autodidacta y con la ayuda de los pares (amigos), así como también, pero con menor frecuencia, recurriendo a algún familiar o a los encargados de los cibercafés. Entre sus destrezas, a diferencia de lo visto para las mujeres, se destacan las habilidades lúdicas ligadas al uso de los videojuegos, y también las técnicas y comunicativas. El acceso a la primera computadora personal y al teléfono celular es relativamente tardío (en comparación con los jóvenes de clase media), y suele ser mediado por la política pública (tanto en el caso de varones como de mujeres de sectores populares).

Los jóvenes y las jóvenes de clase media acceden más tempranamente a las TIC en comparación con los de sectores populares. El primer acceso es tan temprano que, en las entrevistas, con frecuencia les resultaba difícil recordar una fecha particular, señalando que siempre hubo computadora en sus casas, o al menos que la tenían «desde muy chiquitos». Ubicamos el primer acceso significativo en torno de los 5-8 años de edad, realizado casi siempre en el hogar, con computadoras de escritorio o personales de sus padres. Los procesos de aprendizaje nuevamente difieren entre varones y mujeres de clase media: las mujeres señalan a la escuela como un espacio de enseñanza relevante, sumado a la exploración autodidacta con la computadora del hogar, mientras que, para los varones, el aprendizaje se hizo exclusivamente de esta última forma. Alrededor de los 12 años de edad, los y las jóvenes de clase media acceden a su primer teléfono celular y a la primera computadora (puede ser de escritorio o personal), dispositivos comprados por la familia. En cuanto a las habilidades tecnológicas, como ocurría con los jóvenes de sectores populares, las mujeres y los varones se diferencian: las chicas desarrollan destrezas comunicativas y expresivas, mientras que en los chicos prevalecen las destrezas lúdicas, técnicas, operacionales y estratégicas. En este sentido, los varones de clase media muestran, en promedio, un abanico más diverso y complejo de habilidades tecnológicas (en comparación con los varones de sectores populares, que solo excepcionalmente y a través de redes familiares, trayectorias o intereses específicos desarrollan tales habilidades).

La breve comparación que aquí propusimos, en función de material empírico de nuestras investigaciones, busca mostrar algunos de los modos en los cuales género y clase social se intersectan generando desigualdades más o menos intensas. Retornando sobre la perspectiva de las trayectorias biográficas, debemos recordar que estas variaciones se encarnan en articulaciones específicas que, en algunos casos, desafían las generalizaciones. Asimismo, cabe aclarar que la comparación propuesta no se organiza en torno a la valoración de las apropiaciones y usos, sino en términos de ubicar la relación entre las condiciones de acceso, los mecanismos de acción habilitados y las preferencias que objetivamente aparecen materializadas en la relación con las TIC en general.

Como dijimos más arriba, estas preferencias no responden a iniciativas subjetivas individuales, sino que se tejen en tramas complejas de relaciones y contextos. En investigaciones realizadas (Duek, 2016) se identificó el rol de la sociabilidad como organizadora de las elecciones de juego: los espacios y características de los intercambios lúdicos de niños y de niñas se vinculaba con aspiraciones, posiciones en tramas complejas de sentido y con grupos que constituían el núcleo de la cotidianeidad escolar. Jugar no es solamente un juego, sino una forma de vínculo con pares, con espacios y grupos para establecer formas de pertenencia. Las modas y los cambios solo pueden entenderse en tanto se analicen en profundidad las estructuras que contienen las prácticas lúdicas que sostienen niños y niñas. Jugar para pertenecer, jugar para ser: dos resultados claves de la investigación realizada.

En síntesis, la propuesta metodológica planteada permite comprender cómo se vinculan el género y la apropiación de tecnologías en la niñez desde una mirada cualitativa que apunta a identificar en 
profundidad los sentidos de las prácticas. La reconstrucción de trayectorias sirve para detectar si existen diferencias significativas en los procesos de socialización de niños y niñas, que resultarían en trayectorias generizadas en relación con las TIC, tanto en dimensiones objetivas (edad de acceso, patrones de conectividad y de uso, tiempo y frecuencia), como subjetivas (gustos, intereses, habilidades, preferencias e identificaciones). Para ello, el método biográfico resulta adecuado. Asimismo, el análisis comparativo de dichas trayectorias permite captar los procesos por los cuales aquellas diferencias podrían ser moldeadas y experimentadas de maneras diferenciales de acuerdo al sector social de pertenencia. En definitiva, la agenda de investigación es simultáneamente una síntesis de indagaciones teóricas y metodológicas previas y una propuesta que investigadores del campo podrían retomar críticamente en sus propios trabajos (incluso para identificar los errores y fallas que esta agenda pudiera tener). Nos interesó en la investigación (y nos convoca políticamente) comprender cómo las desigualdades de género se intersectan con desigualdades de clase en el caso de la apropiación de TIC.

\section{CONCLUSIONES}

El recorrido que aquí propusimos se ordena en torno de los problemas, las encrucijadas y tensiones que hemos tenido que resolver a lo largo de la última década como investigadores. La agenda que aquí desplegamos tiene como objetivo ordenar algunas cuestiones que consideramos para todas las investigaciones cualitativas del campo. Sin embargo, queremos dejar aquí planteada una serie de conclusiones que quedan abiertas para ser continuadas, cuestionadas o retomadas en la investigación.

En primer lugar, hay que atender una de las confusiones más importantes del campo: no confundir acceso a las TIC con democratización o igualación de posibilidades. En consecuencia, y en segundo lugar, las apropiaciones y usos de las TIC pueden verse en las formas en las que los niños y las niñas juegan. El género opera como diferenciador de gustos, preferencias, páginas y pragmáticas de uso. Las páginas «de nenas» y las «de varones» reproducen los estereotipos, roles y modelos de niños y de niñas que retoman los medios estabilizando imágenes de los más chicos y las prácticas esperables en sus juegos.

En tercer lugar, es fundamental sostener de manera constante tres grandes tareas en las investigaciones: desnaturalizar, analizar, cuestionar. Las tres son herramientas clave para la indagación constante de las innovaciones, las representaciones y las prácticas. Jugar es un nuevo factor clave en la socialización: rankings en redes sociales y en plataformas vinculadas y una constante exhibición de las preferencias y rendimientos mediante «dos clics». Mostrar a otros lo que cada uno hace deviene tarea central para las tramas de sujetos conectados.

En cuarto lugar, consideramos que toda investigación cualitativa debe abordar a los niños y a las niñas como sujetos ubicados en tramas sociales, culturales, políticas y económicas que los configuran y que organizan la relación con su entorno. Componer tecnobiografías es la forma que identificamos como productiva y profunda para identificar las formas en las que aquellas tramas se articulan, vinculan y expanden significativamente.

Acceder a las TIC no supone la inclusión ni tampoco el conocimiento de las pragmáticas de uso. Es necesario demandar, en quinto y último lugar, el reconocimiento de las desigualdades de acceso y uso de TIC en torno del género como un problema que debe ser retomado por parte de los estados. Y es responsabilidad de los investigadores del campo ejercer un constante análisis crítico que no solo alerte sobre estas desigualdades, sino que proponga nuevas miradas y perspectivas para desarmar las representaciones hegemónicas en torno del género, las TIC y las infancias contemporáneas. 


\section{BIBLIOGRAFÍA}

Benítez Larghi, S. (2013). TIC, clase social y género. La constitución de desigualdades sociales y digitales en las juventudes argentinas. En X Jornadas de Sociología de la UBA. Buenos Aires, Argentina.

Berteaux, D. (2005). Le récit de vie. París, Francia: Armand Colin.

Bourdieu, P. (1998). La dominación masculina. Barcelona, España: Anagrama

Cabrera Paz, J. (2009): Profesores Analógicos, Estudiantes Digitales: Una tensión cultural para la gestión pública de las TIC en Educación. En Proceedings of the 3rd ACORN-REDECOM Conference. Ciudad de México, México 22 y 23 de mayo, 2009.

Caillois, R. (1967). Los juegos y los hombres. La máscara y el vértigo. Ciudad de México, México: FCE.

Cassell, J. y Jenkins, H. (1998). From Barbie to mortal kombat. Gender and computer games. Boston, Estados Unidos: MIT Press.

Ching, C. y Vigdor, L. (2005): Technobiographies: Perspectives from Education and the Arts. En First International Congress of Qualitative Inquiry, mayo 2005.

Duek, C. (2013). Infancias entre pantallas. Las nuevas tecnologias y los chicos. Buenos Aires, Argentina: Capital Intelectual.

Duek., C. (2014). Juegos, juguetes y nuevas tecnologias. Buenos Aires, Argentina: Capital Intelectual.

Duek, C. y Enriz, N. (2016). Juegos tradicionales y nuevas tecnologías: Continuidades y apropiaciones. Cadernos de Pesquisa Interdisciplinar em Ciências Humanas, 16(108), 62-74.

Hafkin, N. y Taggart, N. (2001). Gender, information technology and developing countries. Washington, D.C, Estados Unidos: Academy for Educational Development.

Hanish, L. y Fabes, R. (2013). Socialización de género entre pares en niños y niñas de corta edad. En C. L. Martin (Ed.), Género: socialización temprana. Enciclopedia sobre el Desarrollo de la Primera Infancia. Recuperado de: http://www.enciclopedia-infantes.com/Pages/PDF/genero-socializaciontemprana.pdf

Howe, C. y Mercer, N. (2007). Children's social development, peer interaction and classroom learning. Research Survey 2/1b. Cambridge, Inglaterra: University of Cambrigde.

Huizinga, J. (1938). Homo Ludens. Buenos Aires, Argentina: Emecé.

Jenkins, H. (2005). Fans bloggers and gamers exploring participatory culture. Nueva York, Estados Unidos y Londres, Inglaterra: NYU Pres.

Jenson, J. y Castell, S. (2010). Gender, Simulation, and Gaming: Research Review and Redirections. Simulation and gaming, Londres, Inglaterra: Sage.

Kafai, Y.; Heeter, C.; Denner, J. y Sun, J. (2008). Beyond Barbie and Mortal Kombat. New perspectives on gender and gaming. Boston, Estados Unidos: MIT Press.

La Barbera, M. (2010). Género y diversidad entre mujeres. Cuadernos Kóre. Revista de historia y pensamiento de género, 1(2), 55-72. $\quad$ Recuperado de http://kusan.uc3m.es/CIAN/index.php/CK/article/viewFile/1039/480

Leona, P. (s. f.). Hipótesis sobre agenda-setting. Origen, exposición y crítica. En Teorías y prácticas de la comunicación I (Mangone), Facultad de Ciencias Sociales, UBA, Argentina (mimeo).

Livingstone, S. y Helsper, E. (2007). Gradations in digital inclusion: children, young people and the digital divide. New Media and Society, 9(4), 671-696.

Livingstone, S. y Haddon, L. (2012). Theoretical framework for children's internet use. En S. Livingstone, L. Haddon and A. Görzig (Eds.), Children, Risk and Safety on the Internet: Research and policy challenges in comparative perspective (pp. 1-14). Bristol, Inglaterra: The Policy Press.

Lorber, J. (1994). Paradoxes of gender. New Haven, Estados Unidos: Yale University Press.

Morley, D. (1997). Televisión, audiencias y estudios culturales. Buenos Aires, Argentina: Amorrortu.

Prensky, M. (2001). Digital Natives, Digital Immigrants. On the Horizon, NBC Univ. Press, 9(5).

Ridgeway, C.; Backor, K.; Li, E.; Tinkler, J. y Erickson, K. (2009). How Easily Does a Social Difference Become a Status Distinction: Gender Matters. American Sociological Review, 2009, 74 (Feb), 44-62. 
Risman, B. J. y Davis, G. (2013). From Sex Roles to Gender Structure. Current Sociology, 61(5-6): 73355.

Scribano, A. (2008). El proceso de investigación social cualitativo. Buenos Aires, Argentina: Prometeo.

Taylor, S. y Bogdan, R. (1986). Introducción a los métodos cualitativos de investigación. La buisqueda de significados. Buenos Aires, Argentina: Paidós.

Tomte, C. (2008). Return to gender: Gender, ICT and Education. En OECD Expert meeting, Oslo, Noruega 2-3 June 2008.

Turkle, S. (2011). Alone together. Why We Expect More from Technology and Less from Each Other. Boston, Estados Unidos: MIT Press.

Vasilachis De Gialdino, I. (1992). Métodos cualitativos. Buenos Aires: CEAL.

Volman, M. et al. (2005). New technologies, new differences. Gender and ethnic differences in pupils' use of ICT in primary and secondary education. Computers and Education, 45(1), 35-55.

Winocur, R. (2009). Robinson Crusoe ya tiene celular: la conexión como espacio de control de la incertidumbre. Ciudad de México, México: Siglo XXI.

Wolton, D. (1995). Elogio del gran público. Una teoría crítica de la televisión. Barcelona, España: Gedisa.

\section{Fonseca, Journal of Communication}

